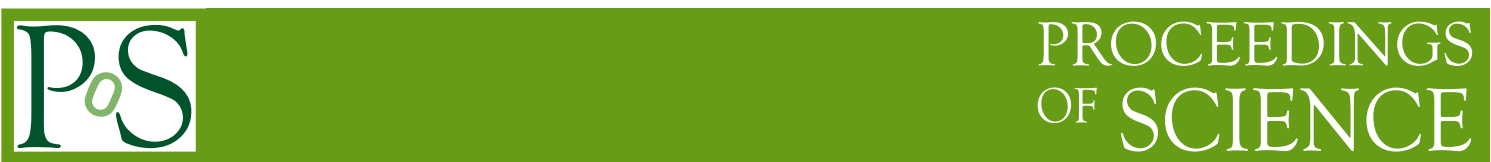

\title{
The NOvA Test Beam Program
}

\section{Michael Wallbank, for the NOvA Collaboration}

University of Cincinnati

E-mail: michael.wallbank@uc.edu

NOvA is a long-baseline oscillation neutrino experiment designed to study and measure a wide range of important topics for neutrino physics such as the neutrino mixing parameters, the neutrino mass hierarchy, and CP violation in the lepton sector. The NOvA Test Beam experiment uses a scaled-down detector of 30 tons to analyze tagged beamline particles. A new tertiary beamline deployed at Fermilab can select and identify electrons, muons, pions, kaons and protons with momenta from 0.2 to $2 \mathrm{GeV} / \mathrm{c}$. Using these data, the Test Beam program will provide NOvA with a better understanding of the largest systematic uncertainties impacting the analyses, including the detector response, calibration, and hadronic and electromagnetic energy resolution. The status and future plans for the NOvA Test beam program, along with preliminary results, are presented in these Proceedings.

40th International Conference on High Energy physics - ICHEP2020

July 28 - August 6, 2020

Prague, Czech Republic (virtual meeting) 


\section{Introduction}

NOvA (NuMI Off-Axis $v_{e}$ Appearance) is a long-baseline neutrino oscillation experiment based at Fermilab in Batavia, Illinois. Neutrinos from the NuMI (Neutrinos from the Main Injector) beam [1] are sampled at two functionally identical detectors: a Near Detector at Fermilab, $1 \mathrm{~km}$ from the beam source, and a Far Detector $810 \mathrm{~km}$ from the beam source located in Ash River, Minnesota.

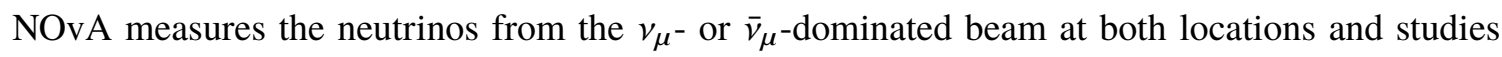
their oscillations as they propagate along the beamline. By searching for $v_{e}$ or $\bar{v}_{e}$ appearance, NOvA aims to resolve the neutrino mass ordering, measure the octant of the mixing angle $\theta_{23}$ and search for evidence of CP-violation in leptons. Additionally, the broad physics program includes measuring neutrino cross-sections at the Near Detector, searching for evidence of sterile neutrinos via non-standard oscillations, and other exotic studies.

The current NOvA 3-flavor oscillation analyses [2] are limited by statistical uncertainties, but as more data are collected these will decrease and the systematic uncertainties will become more limiting. Some of these largest sources of uncertainty include the detector calibration, modeling of the detector response and hadronic and leptonic energy reconstruction. These can be addressed directly using a Test Beam experiment, which can also provide a library of single particles for use in improving the NOvA simulation and reconstruction tools.

\section{The NOvA Test Beam Program}

The NOvA Test Beam Program uses a scaled down NOvA detector with identical technologies to the other NOvA detectors which analyses tagged charged particles from a newly constructed tertiary beamline consisting of protons, pions, muons, electrons and kaons in the 0.2 to $2.0 \mathrm{GeV} / \mathrm{c}$ momentum range relevant to NOvA's neutrino interactions. The detector and beamline were deployed at the Fermilab Test Beam Facility [3] between Summer 2018 and Spring 2019, with commissioning proceeding from May to July 2019 and a first data-taking period from December 2019 to March 2020. A panoramic view of the experiment hall showing the NOvA detector and the tertiary beamline is shown in Figure 1.

\subsection{Beam}

NOvA Test Beam uses the MCenter beam, part of the Fermilab's external beams complex, and is located just downstream of the area previously used by the LArIAT experiment. The beam is derived from protons accelerated up to $120 \mathrm{GeV}$ by the Main Injector and extracted once a minute in a continuous $4.2 \mathrm{~s}$ spill. A secondary beam containing typically $64 \mathrm{GeV}$ protons and pions is created by impinging these primary protons onto a copper target around $120 \mathrm{~m}$ upstream of the NOvA detector. A second target around $14 \mathrm{~m}$ upstream of the NOvA detector is used to produce the tertiary beam containing the lower momentum particles of interest for NOvA Test Beam.

\subsection{Tertiary Beamline}

A full suite of beamline instrumentation provides the trigger for good beam particles, information for particle identification and a precise measurement of their momenta before interactions in the NOvA detector. An analyzer magnet provides a field up to $1.8 \mathrm{~T}$ and is used to select the 


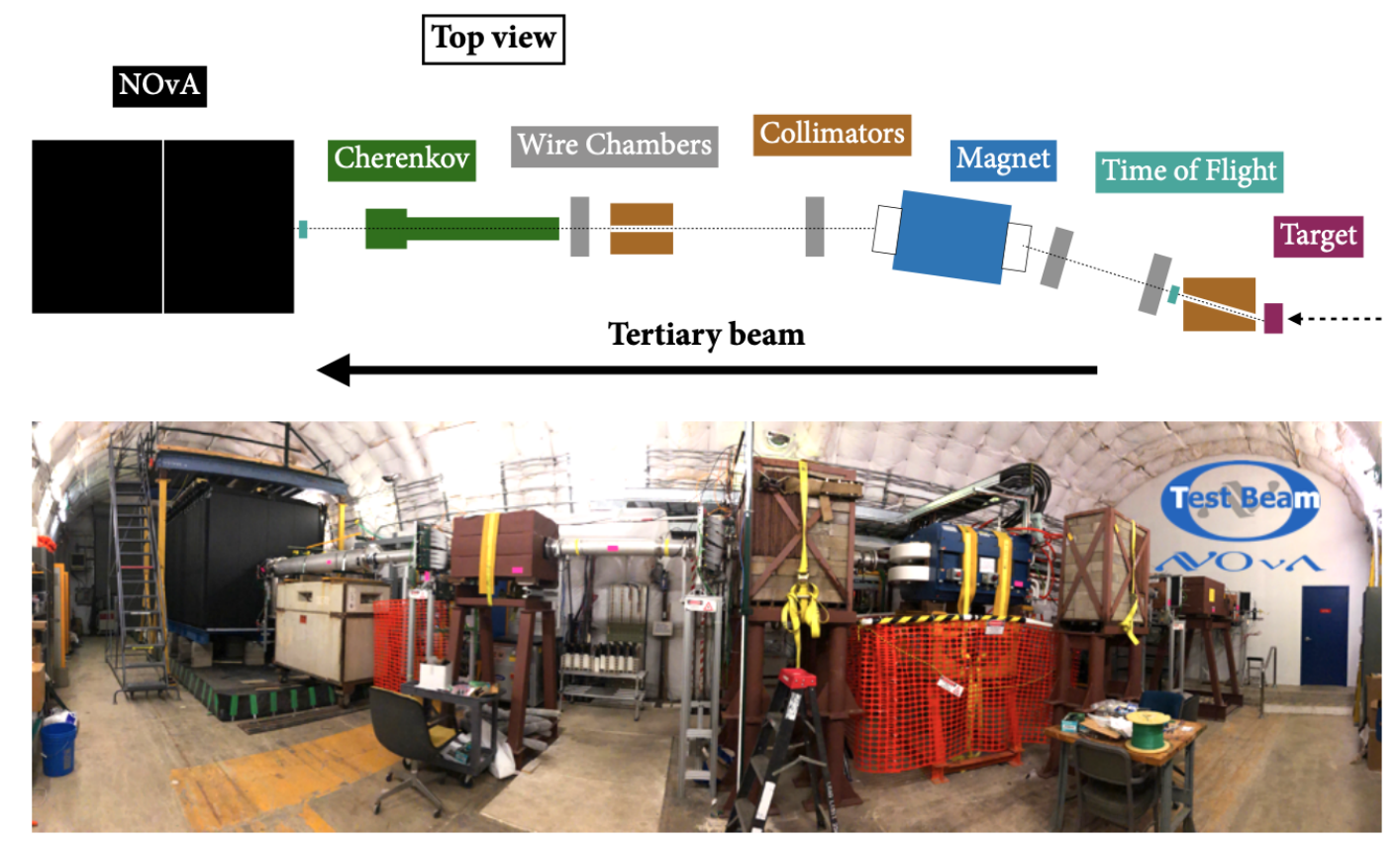

Figure 1: Schematic and panoramic photograph of the NOvA Test Beam experiment. The target is at the far right of the figure and beam travels from right to left. At the left the NOvA detector can be seen. Between, the tertiary beamline is instrumented with a suite of detectors to select the momentum of the beam particles, track them through the beamline and provide information for particle identification. Above the photograph, a schematic shows the main elements of the beamline as a top view.

tertiary beam momentum of interest with tracking provided by four $5.5^{\prime \prime} \times 5.5^{\prime \prime}$ wire chambers with $1 \mathrm{~mm}$ wire pitch, two upstream and two downstream of the magnet. Particle identification is provided by a time-of-flight system consisting of two scintillator arms separated by $13.2 \mathrm{~m}$ to separate particles by mass, and a threshold Cherenkov detector containing $\mathrm{CO}_{2}$ at 1 atm to identify electrons through their emitted Cherenkov radiation. A labeled schematic of the beamline is shown above the photograph in Figure 1.

\subsection{NOvA Detector}

The NOvA detectors are functionally identical and consist of planes of plastic cells [4] filled with an oil-based liquid scintillator [5] and instrumented with wavelength-shifting fibers, read out using an avalanche photo-diode [6]. The planes of cells are orientated alternately horizontal and vertical in the plane perpendicular to the beam to facilitate full 3D particle tracking. The Near (Far) Detector has an active mass of 300 ton (14 kton), and the Test Beam Detector is around an order of magnitude smaller with 30 ton. The Near and Far Detectors use different front-end electronics since they handle different data volumes; the Test Beam Detector is instrumented with both types to facilitate a complete characterization of both NOvA neutrino detectors. A demonstration of the detectors and their technologies is shown in Figure 2. 


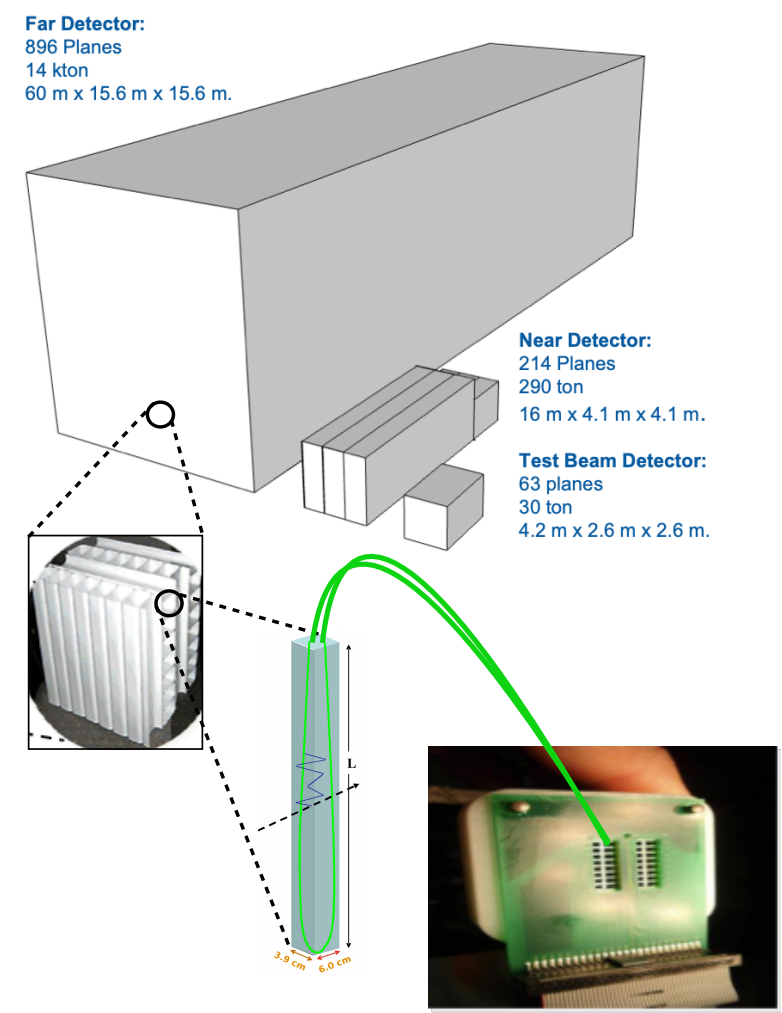

Figure 2: Demonstration of the NOvA detector technology. The cartoons show the three NOvA detectors for comparison of scale. The planes of cells, instrumented with wavelength-shifting fiber, are also shown, along with a photograph of the front-end readout. The technology is functionally identical for each of the detectors.

\section{Preliminary Results}

Figure 3 shows the particle identification capabilities of the beamline detectors using data collected between January 7 and March 20, 2020, corresponding to 5178 selected events, and fully automated reconstruction. These data were collected at two different magnetic fields: $\sim 0.8 \mathrm{~T}$, corresponding to $\sim 1 \mathrm{GeV} / \mathrm{c}$, and $\sim 0.4 \mathrm{~T}$, corresponding to $\sim 500 \mathrm{MeV} / \mathrm{c}$. The heavier protons and kaons are well separated from the lighter electrons, muons and pions, with a further handle on the electrons provided by information from the threshold Cherenkov detector.

These beamline-triggered particles interact in the NOvA detector and are matched together in time. Figure 4 shows a proton candidate particle in the NOvA detector, along with the location of this event on the particle identification plot. The reconstructed time-of-flight for this event is $55.7 \mathrm{~ns}$ and the reconstructed momentum is $1.0 \mathrm{GeV} / \mathrm{c}$, making the particle consistent with a proton. Figure 5 shows an electron candidate, with the characteristic showering in the NOvA detector. The reconstructed time-of-flight for this candidate is $42.7 \mathrm{~ns}$ and the reconstructed momentum is $1.3 \mathrm{GeV} / \mathrm{c}$, which places the particle in the population of lighter particles. There was additionally a signal detected in the threshold Cherenkov detector, implying this is indeed an electron. 


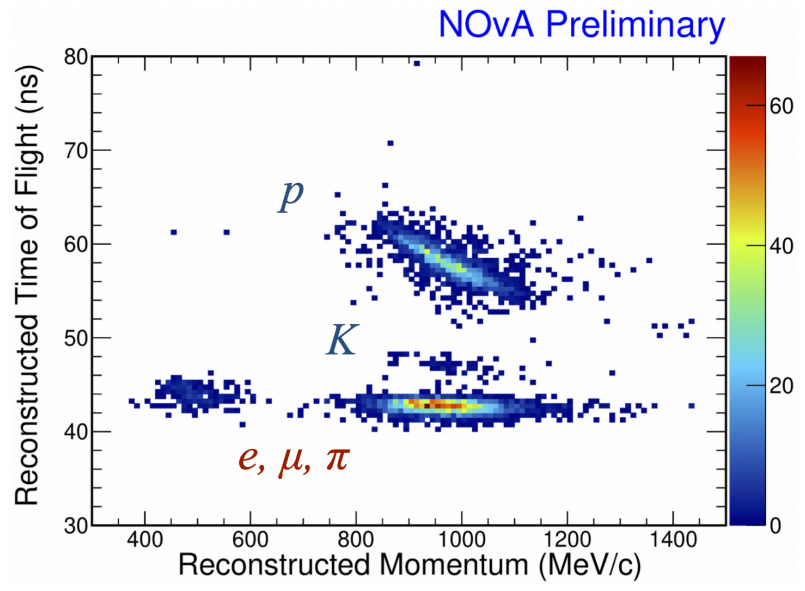

Figure 3: Reconstructed momentum and time-of-flight for tertiary beam particles showing the different populations of particle species. The distributions of protons, kaons, and electrons, muons and pions are labeled.

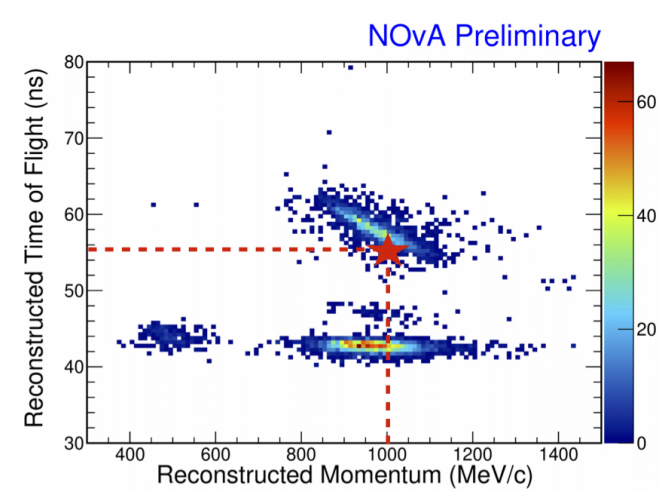

(a) Beamline particle identification.

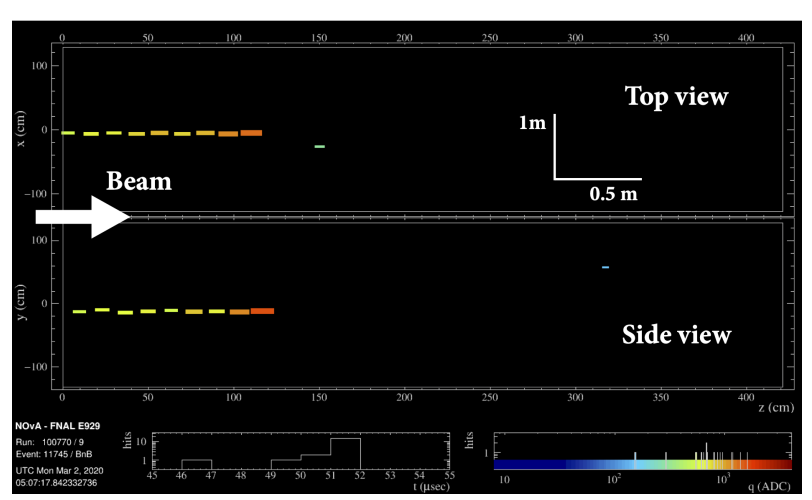

(b) NOvA detector.

Figure 4: Proton candidate identified in the NOvA Test Beam dataset. Figure 4a shows the reconstructed properties of the particles (red star) make it consistent with a proton. The interaction of this particle in the NOvA detector is shown in the event display in Figure 4b. The event display shows the two orthogonal detector views, with each of the pixels representing the light collected on a single cell and the color scale showing the charge. The beam enters at the left and the particle appears to stop and deposit its energy around a third of the way through the detector.

\section{Summary \& Plans}

NOvA has installed, commissioned and operated a new tertiary beamline at the Fermilab Test Beam Facility and collected data using a scaled-down NOvA detector with identical technologies to the Near and Far Detectors. To date, around 6 months of beam data have been collected between May 2019 and March 2020, corresponding to a commissioning phase and a subsequent initial data run. Full detector calibration is ongoing using cosmic interactions and NOvA is beginning the process of analyzing the data with a view to improving the understanding of the NOvA detectors and incorporating improvements into the calibration, reconstruction and analysis.

Recent work over the Summer 2020 shutdown has focused on improving the understanding of 


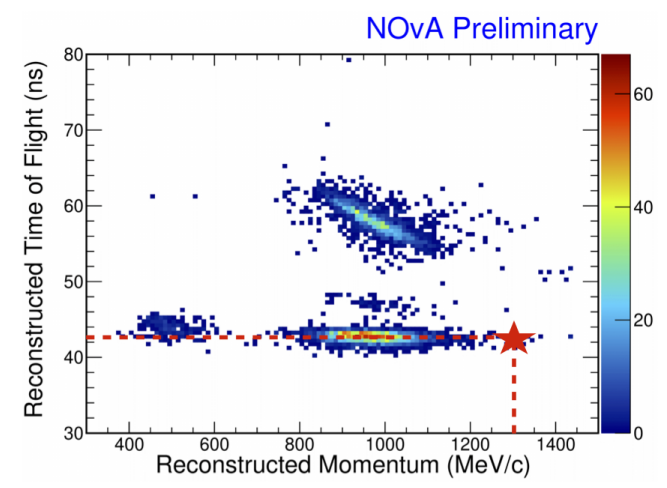

(a) Beamline particle identification.

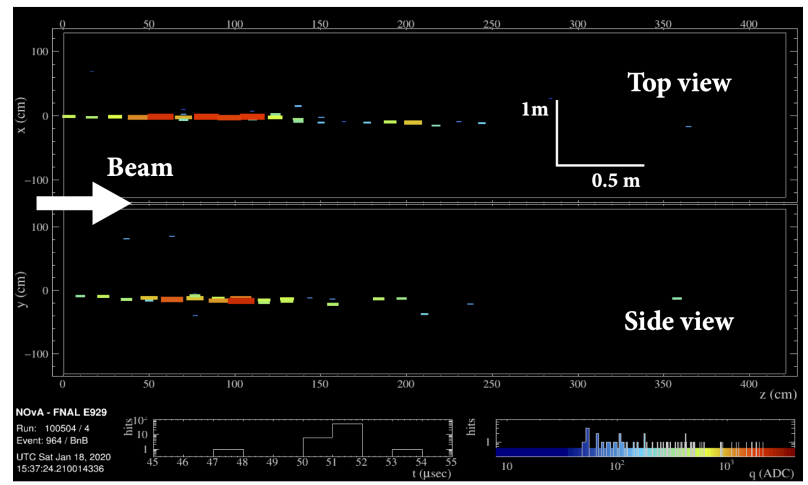

(b) NOvA detector.

Figure 5: Electron candidate identified in the NOvA Test Beam dataset. Figure 5a shows the reconstructed properties of the particles (red star) are consistent with a light particle; additionally, light was detected in the threshold Cherenkov detector, implying an electron. The interaction of this particle in the NOvA detector is shown in the event display in Figure 5b. The event display shows the two orthogonal detector views, with each of the pixels representing the light collected on a single cell and the color scale showing the charge. The beam enters at the left and the characteristic showering of the electromagnetic interactions of the electron candidate can be seen.

the beam using data collected in the previous period and simulations to identify improvements for future data-taking. Further operations are planned during the next Fermilab beam period between December 2020 and Summer 2021 to augment the dataset with data collected under improved operational conditions.

\section{References}

[1] P. Adamson et al., Nucl. Instr. Methods Phys. Res., Sect A 806, 279 (2016).

[2] M. A. Acero et al. (The NOvA Collaboration), Phys. Rev. Lett. 123, 15, 151803 (2009).

[3] Fermilab Test Beam Facility (FTBF), http://ftbf.fnal.gov.

[4] R. L. Talaga et al., Nucl. Instrum. Methods Phys. Res., Sect A 861, 77 (2017).

[5] S. Mufson et al., "Nucl. Instrum. Methods Phys. Res., Sect A 799, 1 (2015).

[6] The NOvA Avalanche Photodiode is a custom variant of the Hamamatsu S8550, https://www.hamamatsu.com/us/en/product/type/S8550-02/index.html 